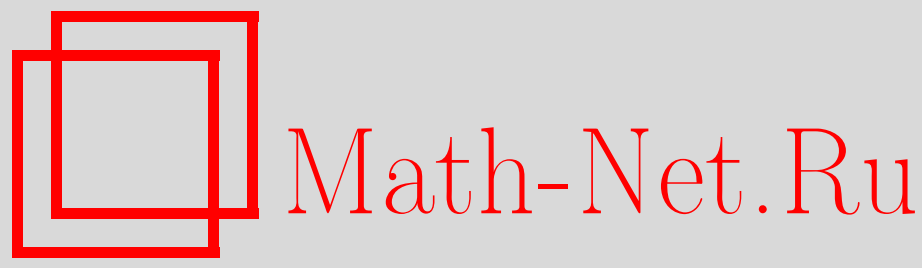

П. А. Путров, Интеграл по траекториям в энергетическом представлении в квантовой механике, ТМ $\Phi, 2008$, том 156, номер 1, 92-111

DOI: https://doi.org/10.4213/tmf6232

Использование Общероссийского математического портала Math-Net.Ru подразумевает, что вы прочитали и согласны с пользовательским соглашением http://www.mathnet.ru/rus/agreement

Параметры загрузки:

IP: 54.196.121.252

26 апреля 2023 г., 08:01:58

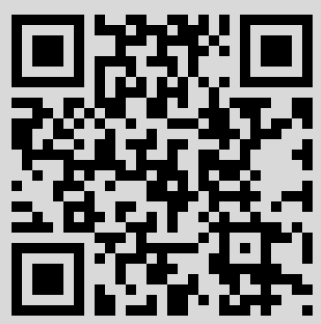




\title{
ИНТЕГРАЛ ПО ТРАЕКТОРИЯМ В ЭНЕРГЕТИЧЕСКОМ ПРЕДСТАВЛЕНИИ В КВАНТОВОЙ МЕХАНИКЕ
}

\begin{abstract}
Рассматривается альтернативный подход к квантовой механике, использующий интеграл по траекториям. Представлена резольвента гамильтониана (являющаяся преобразованием Лапласа оператора эволюции) в форме, которая имеет смысл суммы по путям, но определена значительно лучше, чем обычный функциональный интеграл. Это представление исследуется с различных сторон, и такой подход к квантовой механике сравнивается со стандартными.
\end{abstract}

Ключевые слова: интеграл по траекториям, квантовая механика, инстантоны.

\section{1. ВВЕДЕНИЕ}

Данную работу можно рассматривать как попытку получить математически хорошо определенное представление амплитуды перехода в квантовой механике через сумму по траекториям. Представление, которое мы получаем, является рядом, каждый член которого содержит конечное число интегрирований. Пространство $\operatorname{Paths}\left(x^{\prime}, x^{\prime \prime}\right)$, которое мы определяем и по которому мы интегрируем, намного проще пространства траекторий $\Gamma\left(x^{\prime}, x^{\prime \prime}\right)=\left\{x(\cdot) \in C^{1}([0, T], V) \mid x(0)=x^{\prime}, x(T)=x^{\prime \prime}\right\}$, фигурирующего в обычном подходе [1] через интеграл по траекториям. Мы определяем подходящую форму объема и функционал действия на этом пространстве и используем эти объекты для написания интеграла по траекториям.

Получаемое представление может помочь понять, какие именно траектории вносят основной вклад в разных ситуациях.

Структура работы следующая. Раздел 2 содержит основные определения и главный результат. В разделе 3 мы получаем интересную связь между суммой по определенному подмножеству всех путей и решением уравнения Шредингера, а также даем альтернативное доказательство нашего представления. В разделе 4 рассматривается специальный тип потенциалов (ступенчатые потенциалы), для которых наше представление сильно упрощается. Разделы 5 и 6 содержат рассмотрение физических приложений и соответствия некоторым обычным методам в квантовой механике. Они написаны не в строгом математическом стиле. В разделе 7 мы даем

${ }^{*}$ Санкт-Петербургский государственный университет, Санкт-Петербург, Россия. E-mail: putrov@itep.ru 
набросок возможного обобщения (возможно, не лучшего) на $D$-мерную квантовую механику. Наконец, в разделе 8 обсуждаются возможные направления дальнейших исследований.

\section{2. АЛЬТЕРНАТИВНОЕ ПРЕДСТАВЛЕНИЕ АМПЛИТУДЫ ПЕРЕХОДА}

Мы будем работать в одномерной квантовой механике (с евклидовым временем) с таргет-пространством ${ }^{1)} V=\mathbb{R}$. В качестве координат на $V$ будем использовать переменные $x$. Будем рассматривать гамильтониан, действующий в гильбертовом пространстве $L_{2}(V)$, в форме $H=-\hbar^{2} \partial^{2} / 2+U(x)$, где потенциал является функцией $U \in C^{1}(V, \mathbb{R}) \quad(U(x) \rightarrow+\infty, x \rightarrow \pm \infty)$, а $\partial \equiv d / d x$. Центральный объект наших исследований - оператор эволюции в энергетическом представлении $K_{E}$. В обычном (или "временно́м") представлении это $K_{T}=e^{-H T / \hbar}$. Под энергетическим представлением мы имеем в виду преобразование Лапласа, т.е. функции времени $T$ переходят в функции энергии $E$, а именно

$$
K_{E}=\int_{0}^{+\infty} d T K_{T} e^{E T / \hbar}=\frac{\hbar}{H-E} .
$$

Мы всегда будем предполагать, что $E$ не совпадает ни с одним из собственных значений $H$. При рассмотрении соответствующих ядер будем просто указывать зависимость от переменных $x$ :

$$
K_{T}\left(x^{\prime \prime}, x^{\prime}\right)=\left\langle x^{\prime \prime}\left|e^{-H T / \hbar}\right| x^{\prime}\right\rangle, \quad K_{E}\left(x^{\prime \prime}, x^{\prime}\right)=\left\langle x^{\prime \prime}\left|\frac{\hbar}{H-E}\right| x^{\prime}\right\rangle .
$$

В нашем подходе удобнее работать с функцией $p(x)=\sqrt{2(E-U(x))}$. Ветвь корня следует выбирать так, что $\operatorname{Im}[p(x)]>0$. Отметим, что мы не указываем явно зависимость $p(x)$ от $E$. Положим для упрощения $\hbar=1$ (в дальнейшем, однако, мы восстановим ее в некоторых соотношениях). Тогда

$$
K_{E}=-\frac{2}{\partial^{2}+p(x)^{2}} .
$$

Введем оператор

$$
\Phi=\frac{1}{\sqrt{p}} \frac{i}{p+i \partial} \frac{1}{\sqrt{p}}
$$

с ядром

$$
\Phi\left(x^{\prime \prime}, x^{\prime}\right)=\frac{1}{\sqrt{p\left(x^{\prime \prime}\right) p\left(x^{\prime}\right)}} \exp \left\{i \int_{x^{\prime}}^{x^{\prime \prime}} p(\xi) d \xi\right\} \theta\left(x^{\prime \prime}-x^{\prime}\right) .
$$

Это квазиклассическая амплитуда перехода в правом направлении. Транспонированный оператор имеет вид

$$
\Phi^{\mathrm{T}}=\frac{1}{\sqrt{p}} \frac{i}{p-i \partial} \frac{1}{\sqrt{p}},
$$

\footnotetext{
1) По умолчанию мы рассматриваем $\mathbb{R}^{1}$, но можно работать также с другой топологией (например, рассматривать отрезок или $\left.S^{1}\right)$. Общие итоги будут такими же.
} 


$$
\Phi^{\mathrm{T}}\left(x^{\prime \prime}, x^{\prime}\right)=\frac{1}{\sqrt{p\left(x^{\prime \prime}\right) p\left(x^{\prime}\right)}} \exp \left\{i \int_{x^{\prime \prime}}^{x^{\prime}} p(\xi) d \xi\right\} \theta\left(x^{\prime}-x^{\prime \prime}\right) .
$$

Это квазиклассическая амплитуда перехода в левом направлении. Заметим, что ядра конечны при больших $x$ из-за того, что $\operatorname{Im}[p(x)]>0$.

ОпРЕДЕЛЕНиЕ 1. Диаграммные правила для операторов определяются следующим образом: для пропагаторов вперед (направо) и назад (налево)

$$
x \uparrow \Phi=\ell, \quad \Phi^{\mathrm{T}}=\nmid
$$

для точек отражения

$$
\uparrow p^{\prime} / 2=\bigvee, \quad-p^{\prime} / 2=\Lambda
$$

где $p^{\prime} \equiv \partial p / \partial x$.

Для того чтобы написать выражение, соответствующее определенной диаграмме, нужно перемножить операторы, соответствующие всем элементам, в порядке, определенном стрелками.

ЛЕмма 1. Имеет место следующее разложение:

$$
\begin{aligned}
& -i K_{E}=\Phi+\Phi^{\mathrm{T}}-\Phi^{\mathrm{T}} \frac{p^{\prime}}{2} \Phi+\Phi \frac{p^{\prime}}{2} \Phi^{\mathrm{T}}-\Phi^{\mathrm{T}} \frac{p^{\prime}}{2} \Phi \frac{p^{\prime}}{2} \Phi^{\mathrm{T}}-\Phi \frac{p^{\prime}}{2} \Phi^{\mathrm{T}} \frac{p^{\prime}}{2} \Phi+\cdots= \\
& =f+\downarrow+\hat{\gamma}+\boldsymbol{\gamma}+\hat{\gamma}+\ldots
\end{aligned}
$$

ДокАзАтельство. Разложение может быть явно записано как

$$
\begin{aligned}
\frac{2 i}{p^{2}+\partial^{2}}= & \frac{1}{\sqrt{p}} \frac{i}{p+i \partial} \frac{1}{\sqrt{p}}+\frac{1}{\sqrt{p}} \frac{i}{p-i \partial} \frac{1}{\sqrt{p}}+ \\
& +\frac{1}{\sqrt{p}} \frac{i}{p+i \partial} \frac{p^{\prime}}{2 p} \frac{i}{p-i \partial} \frac{1}{\sqrt{p}}-\frac{1}{\sqrt{p}} \frac{i}{p-i \partial} \frac{p^{\prime}}{2 p} \frac{i}{p+i \partial} \frac{1}{\sqrt{p}}+\cdots .
\end{aligned}
$$

Ряды (8), (9) можно просуммировать явно, потому что они состоят из геометрических прогрессий типа $1 /(A-B)=A^{-1}+A^{-1} B A^{-1}+\cdots$. Для того чтобы доказать формулу (9), рассмотрим операторы $u_{+}$и $u_{-}$, которые являются суммами членов правой части, начинающихся соответственно с $\Phi$ и $\Phi^{\mathrm{T}}$. Они удовлетворяют следующим рекуррентным соотношениям:

$$
(p \pm i \partial) u_{ \pm}=\frac{i}{p}-i \frac{p^{\prime}}{2 p}\left(u_{+}-u_{-}\right) .
$$

В терминах $u=u_{+}+u_{-}$и $w=u_{+}-u_{-}$эти соотношения можно переписать как

$$
p u+i \partial w=\frac{2 i}{p}-\frac{i p^{\prime}}{p} w, \quad p w+i \partial u=0 .
$$

Затем, исключая $w$, получаем требуемый результат:

$$
\left(p^{2}+\partial^{2}\right) u=2 i \Rightarrow u=-i K_{E} .
$$


В приложении А показано, как представление (9) согласуется с пертурбативным разложением для $K_{E}$. Каждая диаграмма в формуле (8) соответствует в некотором смысле схеме движения частицы. Таким образом, в этом уже можно увидеть сумму по траекториям. Рассмотрим это более систематически.

ОПредЕЛЕНИЕ 2. Пусть $\Delta \subset V$. Тогда определим пространство $\operatorname{Paths}_{\Delta}^{N}\left(x^{\prime}, x^{\prime \prime}\right) \subset$ $\left\{x^{\prime}\right\} \times \Delta^{N} \times\left\{x^{\prime \prime}\right\}$ (или $\left.\operatorname{Paths}_{\Delta}^{N}: V \times V \rightarrow 2^{\Delta^{N}}\right)$ :

$$
\begin{aligned}
& \operatorname{Paths}_{\Delta}^{N}\left(x^{\prime}, x^{\prime \prime}\right)=\left\{\left(x_{0} \equiv x^{\prime}, x_{1}, \ldots, x_{N}, x_{N+1} \equiv x^{\prime \prime}\right) \mid x_{i} \in \Delta,\right. \\
& \left.\quad\left(x_{i+1}-x_{i}\right)\left(x_{i}-x_{i-1}\right)<0,1 \leqslant i \leqslant N\right\}, \\
& \operatorname{Paths}^{N}\left(x^{\prime}, x^{\prime \prime}\right) \equiv \operatorname{Paths}_{V}^{N}\left(x^{\prime}, x^{\prime \prime}\right) \supset \operatorname{Paths}_{\Delta}^{N}\left(x^{\prime}, x^{\prime \prime}\right) \quad \forall \Delta, \\
& \operatorname{Paths}_{\Delta}\left(x^{\prime}, x^{\prime \prime}\right)=\bigsqcup_{N=0}^{\infty} \operatorname{Paths}_{\Delta}^{N}\left(x^{\prime}, x^{\prime \prime}\right) .
\end{aligned}
$$

ЗАмечание 1 . По умолчанию будем обозначать элемент $P \in \operatorname{Paths}\left(x^{\prime}, x^{\prime \prime}\right)$ следующим образом:

$$
P=\left(x_{0} \equiv x^{\prime}, x_{1}, \ldots, x_{N}, x_{N+1} \equiv x^{\prime \prime}\right) \in \operatorname{Paths}^{N}\left(x^{\prime}, x^{\prime \prime}\right) \subset \operatorname{Paths}\left(x^{\prime}, x^{\prime \prime}\right) .
$$

Мы будем всегда отождествлять $x_{0} \equiv x^{\prime}, x_{N+1} \equiv x^{\prime \prime}$ в подобных случаях, не оговаривая это отдельно. Будем называть $x_{k}, 1 \leqslant k \leqslant N$, точками отражения, $x^{\prime}-$ начальной точкой, а $x^{\prime \prime}-$ конечной точкой пути $P$.

ОпредЕлЕниЕ 3 . Определим знаковую функцию $\rightleftarrows(\cdot) \cdot \operatorname{Paths}^{N} \times\{1, \ldots, N\} \rightarrow$ $\mathbb{Z}_{2}$ :

$$
\rightleftarrows{ }_{k}^{(P)}=\theta\left(x_{k}-x_{k-1}\right) .
$$

ОПРедЕЛЕНиЕ 4. Определим функцию $S: \operatorname{Paths}\left(x^{\prime}, x^{\prime \prime}\right) \rightarrow \mathbb{C}$ :

$$
S[P]=-\sum_{k=0}^{N}(-1)_{k+1}^{\rightleftarrows_{k+1}^{(P)}} \int_{x_{k}}^{x_{k+1}} p(\xi) d \xi \equiv \int_{P} p(\xi) d \xi,
$$

т.е. интеграл от $p(\xi)$ такой, что каждый член имеет положительную мнимую часть.

ЗАмечание 2. $S[P]$ - укороченное действие, вычисленное на пути $P$ по классической формуле.

ОпредЕлениЕ 5. Сначала определим форму “объема"2) на $\operatorname{Paths}^{N}\left(x^{\prime}, x^{\prime \prime}\right)$ :

$$
\Omega_{N}=\bigwedge_{k=1}^{N} d x_{k} \frac{p^{\prime}\left(x_{k}\right)}{2 p\left(x_{k}\right)}(-1)^{\rightleftarrows_{k}} .
$$

Тогда форму объема на $\operatorname{Paths}\left(x^{\prime}, x^{\prime \prime}\right)$ можно определить просто как их сумму:

$$
\Omega=\sum_{N=0}^{\infty} \Omega_{N}
$$

2) Мы используем кавычки, потому что эта форма, конечно, не обязательно положительно определена. 
Теперь несложно переписать выражение (8) в лемме 1 в терминах ядер операторов и получить следующую теорему.

Теорема 1. Имеет место следующее выражение:

$$
\left\langle x^{\prime \prime}\left|\frac{1}{H-E}\right| x^{\prime}\right\rangle=\frac{1}{\sqrt{p\left(x^{\prime}\right) p\left(x^{\prime \prime}\right)}} \int_{\operatorname{Paths}\left(x^{\prime}, x^{\prime \prime}\right)} \Omega e^{i S},
$$

или, более явно,

$$
\begin{aligned}
& \left\langle x^{\prime \prime}\left|\frac{1}{H-E}\right| x^{\prime}\right\rangle= \\
& \quad=\frac{1}{\sqrt{p\left(x^{\prime}\right) p\left(x^{\prime \prime}\right)}} \sum_{N=0}^{\infty} \int_{\text {Paths }^{N}\left(x^{\prime}, x^{\prime \prime}\right)} \prod_{k=1}^{N} d x_{k} \frac{p^{\prime}\left(x_{k}\right)}{2 p\left(x_{k}\right)}(-1)^{\rightleftarrows} \exp \left\{i \int_{P} p(\xi) d \xi\right\} .
\end{aligned}
$$

ЗАмечание 3 . Сумма по $N$ соответствует сумме в выражении (8). $N$-й член в сумме (22) содержит два члена из суммы (8), при этом $N$ является количеством множителей $p^{\prime}$, входящих в них. Свертка по точкам $\left\{x_{k}\right\}_{k=1}^{N}$ производится по множеству $\left\{\left(x_{i+1}-x_{i}\right)\left(x_{i}-x_{i-1}\right)<0\right\}$. Эти неравенства возникают из-за наличия $\theta$-функций в ядрах (5) и (7). Экспонента в сумме (22) есть произведение экспонент, входящих в ядра операторов $\Phi$ и $\Phi^{\mathrm{T}}$.

Это согласуется с нашими диаграммными правилами из определения 1.

ОПРЕДЕлЕНиЕ 6. Диаграммные правила для суммы (22) определим следующим образом: пропагаторы

$$
x \uparrow \quad \int_{x_{i}}^{x_{i+1}} \longrightarrow \exp \left[i \int_{x_{i}}^{x_{i+1}} p(\xi) d \xi\right], \quad \backslash_{x_{i+1}}^{x_{i}} \exp \left[i \int_{x_{i+1}}^{x_{i}} p(\xi) d \xi\right] ;
$$

точки отражения

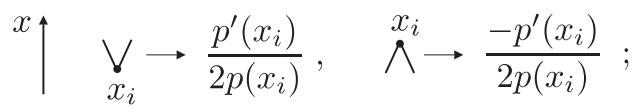

начальные и конечные точки

$$
\underset{x^{\prime}}{x} \rightarrow p^{-1 / 2}\left(x^{\prime}\right), \quad \stackrel{x^{\prime \prime}}{\longrightarrow} \rightarrow p^{-1 / 2}\left(x^{\prime \prime}\right) .
$$

Также следует проинтегрировать по положениям точек отражения таким образом, чтобы структура стрелок при этом не изменялась (т.е. количество стрелок и их ориентация оставались такими же).

Тогда сумму (22) можно записать в виде

$$
\left\langle x^{\prime \prime}\left|\frac{1}{H-E}\right| x^{\prime}\right\rangle=x^{\prime} \mid
$$


СледСТВиЕ 1. Имеет место следующее представление для статистической суммы:

$$
Z_{E}=\operatorname{Tr} \frac{1}{H-E}=\int_{V} \frac{d x}{p(x)} \int_{\operatorname{Paths}(x, x)} \Omega e^{i S} .
$$

\section{3. СВЯЗЬ С УРАВНЕНИЕМ ШРЕДИНГЕРА}

Рассмотрим функцию

$$
I_{\mathrm{R}}(x)=\int_{\operatorname{Paths}_{[x,+\infty)}(x, x)} \Omega e^{i S},
$$

т.е. сумму по путям с точками отражения, лежащими по правую сторону от $x$ :

$$
I_{R}(x) \equiv n^{-1}
$$

Рассматривая инфинитезимально малый сдвиг $x$, можно получить дифференциальное уравнение для $I_{\mathrm{R}}(x)$ :

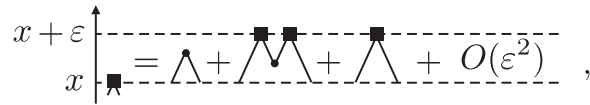

$$
\begin{aligned}
& \frac{d I_{\mathrm{R}}}{d x}=\frac{p^{\prime}}{2 p}\left(1-I_{\mathrm{R}}^{2}\right)-i \frac{2 p}{\hbar} I_{\mathrm{R}} .
\end{aligned}
$$

Аналогично для функции

$$
I_{\mathrm{L}}(x)=\int_{\operatorname{Paths}_{(-\infty, x]}(x, x)} \Omega e^{i S}
$$

имеем

$$
\frac{d I_{\mathrm{L}}}{d x}=\frac{p^{\prime}}{2 p}\left(1-I_{\mathrm{L}}^{2}\right)+i \frac{2 p}{\hbar} I_{\mathrm{L}}
$$

Следует добавить очевидные граничные условия для этих уравнений ${ }^{3)}:\left.I_{\mathrm{L}}\right|_{-\infty}=0$, $\left.I_{\mathrm{R}}\right|_{+\infty}=0$. В уравнениях $E$ играет роль параметра.

Производя простые замены переменных, несложно получить следующий результат.

ПредЛОЖЕНИЕ. Уравнения (27) и (25) эквивалентны уравнению Шредингера. А именно

$$
I_{\mathrm{R}}=-\frac{\left(\ln \psi_{\mathrm{R}}\right)^{\prime}-i p / \hbar}{\left(\ln \psi_{\mathrm{R}}\right)^{\prime}+i p / \hbar}, \quad I_{\mathrm{L}}=-\frac{\left(\ln \psi_{\mathrm{L}}\right)^{\prime}+i p / \hbar}{\left(\ln \psi_{\mathrm{L}}\right)^{\prime}-i p / \hbar}
$$

где $\psi_{\mathrm{L}}, \psi_{\mathrm{R}}$ суть решения уравнения Шредингера

$$
\psi^{\prime \prime}=-\frac{p^{2}}{\hbar^{2}} \psi, \quad p(x)^{2}=2(E-U(x)),
$$

с граничными условиями $\left.{ }^{4)} \psi_{\mathrm{L}}\right|_{-\infty}=0,\left.\psi_{\mathrm{R}}\right|_{+\infty}=0$.

\footnotetext{
3) Для случая, когда $V=[a, b]$, их следует заменить на $\left.I_{\mathrm{L}}\right|_{a}=-1,\left.I_{\mathrm{R}}\right|_{b}=-1$.

4) Если $V=[a, b]$, их следует заменить на $\left.\psi_{\mathrm{L}}\right|_{a}=0,\left.\psi_{\mathrm{R}}\right|_{b}=0$.
}

4 Теоретическая и математическая физика, т. 156, № 1, 2008 г. 
$\mathrm{C}$ помощью $I_{\mathrm{L}}$ и $I_{\mathrm{R}}$ легко записать сумму по путям для статистической суммы:

$$
\int_{\operatorname{Paths}(x, x)} \Omega e^{i S}=\frac{1+I_{\mathrm{L}}(x)+I_{\mathrm{R}}(x)+I_{\mathrm{L}}(x) I_{\mathrm{R}}(x)}{1-I_{\mathrm{L}}(x) I_{\mathrm{R}}(x)} .
$$

Для этого нужно лишь выполнить суммирование (геометрической прогрессии) в точке $x$.

Введем вронскиан пары $\left(\psi_{\mathrm{L}}, \psi_{\mathrm{R}}\right): W(E)=\left(\psi_{\mathrm{L}}^{\prime}(x) \psi_{\mathrm{R}}(x)-\psi_{\mathrm{R}}^{\prime}(x) \psi_{\mathrm{L}}(x)\right)$, имеющий нули в собственных значениях $H$. Подставляя $(28)$ в $(30)$ и используя то, что

$$
W^{\prime}(E) \equiv \frac{d W(E)}{d E}=2 \int_{V} \psi_{\mathrm{L}}(x) \psi_{\mathrm{R}}(x) d x,
$$

получим, что

$$
\int_{V} \frac{d x}{p(x)} \int_{\operatorname{Paths}(x, x)} \Omega e^{i S}=-\frac{W^{\prime}(E)}{W(E)}=\operatorname{Tr} \frac{1}{H-E}=Z_{E} .
$$

Это вычисление можно рассматривать как альтернативное доказательство выражения (23).

Следовательно, если мы знаем решения уравнения Шредингера, то мы знаем $I_{\mathrm{R}}$ и $I_{\mathrm{L}}$, которые содержат информацию о траекториях, дающих вклад в (23). Производные $I_{\mathrm{R}}$ и $I_{\mathrm{L}}$ дают нам информацию о траекториях, отражающихся в окрестности $x$.

Аналогичные вычисления (их можно найти в приложении Б) можно сделать и для амплитуды перехода. В этом случае нам следует ввести также функцию $J_{\mathrm{R}}\left(x, x^{\prime \prime}\right)$ (или $J_{\mathrm{L}}\left(x, x^{\prime}\right)$ ) для суммы по всем траекториям, соединяющим $x$ и $x^{\prime \prime}$ и лежащим справа от $x$ :

$$
J_{\mathrm{R}}\left(x, x^{\prime \prime}\right)=\int_{\operatorname{Paths}_{[x,+\infty)}\left(x, x^{\prime \prime}\right)} \Omega e^{i S} .
$$

Она удовлетворяет следующему уравнению:

$$
J_{\mathrm{R}}^{\prime}\left(x, x^{\prime \prime}\right)=-i \frac{p}{\hbar} J_{\mathrm{R}}\left(x, x^{\prime \prime}\right)-\frac{p^{\prime}}{2 p} J_{\mathrm{R}}\left(x, x^{\prime \prime}\right) I_{\mathrm{R}}(x), \quad x<x^{\prime \prime},
$$

с граничным условием

$$
J_{\mathrm{R}}\left(x^{\prime \prime}, x^{\prime \prime}\right)=I_{\mathrm{R}}\left(x^{\prime \prime}\right)+1 .
$$

Используя эту функцию, можно получить

$$
\begin{aligned}
& \frac{1}{\sqrt{p\left(x^{\prime}\right) p\left(x^{\prime \prime}\right)}} \int_{\operatorname{Paths}\left(x, x^{\prime \prime}\right)} \Omega e^{i S[P]}=-2 \frac{\psi_{\mathrm{L}}\left(x^{\prime}\right) \psi_{\mathrm{R}}\left(x^{\prime \prime}\right)}{W(E)}= \\
& =\sum_{n} \frac{\psi_{n}\left(x^{\prime}\right) \psi_{n}\left(x^{\prime \prime}\right)}{E_{n}-E}=\left\langle x^{\prime}\left|\frac{1}{H-E}\right| x^{\prime \prime}\right\rangle=K_{E}\left(x^{\prime}, x^{\prime \prime}\right)
\end{aligned}
$$

для $x^{\prime}<x^{\prime \prime}$. Это альтернативное доказательство выражений (21), (22).

Отметим еще одно следствие. Если требуется вычислить интеграл по траекториям таким, что $x$ не выходит из некоторого фиксированного отрезка, можно получить соответствующее ядро из решений уравнения Шредингера с граничными условиями $\partial \ln \psi / \partial x= \pm i p / \hbar$ (такими, что $\left.I_{\mathrm{L}, \mathrm{R}}=0\right)$. Это отличается от стандартного случая с бесконечно высокими потенциальными стенками на концах отрезка, для которого нам следует положить $\psi=0$ на границе $\left(I_{\mathrm{L}, \mathrm{R}}=-1\right)$. 


\section{4. СТУПЕНЧАТЫЕ ПОТЕНЦИАЛЫ}

Легко заметить, что из-за наличия множителей $p^{\prime} /(2 p)$ интегралы в выражении (22) сильно упрощаются в областях с постоянным потенциалом. Поэтому интересно рассмотреть ступенчатый потенциал $U(x)$ с $U(x)=U_{j}, x \in \Delta_{j} \subset V \quad\left(\Delta_{j}=\right.$ $\left.\left[y_{j-1}, y_{j}\right]\right)$ (т.е. $U(x)$ имеет разрывы в точках $\left.y_{j} \quad\left(y_{j}>y_{j+1}\right), j \in \mathbb{Z}\right)$. Обозначим длину интервала $\Delta_{j}$ через $a_{j}$ и $p_{j}=\sqrt{2\left(E-U_{j}\right)}$.

Нам потребуется найти интеграл в окрестности точки $y_{j}$, где потенциал изменяется с $U_{j}$ до $U_{j+1}$. Для этого регуляризуем "ступеньку", а именно положим, что в интервале $\Sigma_{\epsilon}=\left[y_{j}-\epsilon, y_{j}+\epsilon\right]$ имеется некоторая гладкая функция $p(x)=\sqrt{2(E-U(x))}$ такая, что $p\left(y_{j}-\epsilon\right)=p_{j}, p\left(y_{j}+\epsilon\right)=p_{j+1}$. Рассмотрим сначала интеграл, соответствующий случаю, когда траектория как-либо отражается от этой ступеньки. Тогда нас интересует величина

$$
-\alpha_{j}=\lim _{\epsilon \rightarrow 0} \int_{\operatorname{Paths}_{\Sigma_{\epsilon}}\left(y_{j}-\epsilon, y_{j}-\epsilon\right)} \Omega e^{i S / \hbar}=\int_{\operatorname{Paths}_{\Sigma_{\epsilon}}\left(y_{j}-\epsilon, y_{j}-\epsilon\right)} \Omega,
$$

потому что последнее выражение, очевидно, не зависит от $\epsilon$. Заметим, что это выражение можно рассматривать как предел при $\hbar \rightarrow \infty$ (так как мы используем то, что $\left.e^{i S / \hbar} \rightarrow 1\right)$; таким образом, ступенька является бесконечно квантовой системой (в отличие от областей постоянного потенциала, которые, наоборот, можно рассматривать классически), и в интеграле по Paths остается лишь форма объема $\Omega$. Приведенные в приложении В вычисления дают

$$
\alpha_{j}=\frac{p_{j+1}-p_{j}}{p_{j+1}+p_{j}}
$$

Аналогично можно получить, что

$$
\begin{gathered}
\lim _{\epsilon \rightarrow 0} \int_{\operatorname{Paths}_{\Sigma_{\epsilon}}\left(y_{j}+\epsilon, y_{j}+\epsilon\right)} \Omega e^{i S / \hbar}=+\alpha_{j}, \\
\lim _{\epsilon \rightarrow 0} \int_{\operatorname{Paths}_{\Sigma_{\epsilon}}\left(y_{j}-\epsilon, y_{j}+\epsilon\right)} \Omega e^{i S / \hbar}=1-\alpha_{j}, \quad \lim _{\epsilon \rightarrow 0} \int_{\operatorname{Paths}_{\Sigma_{\epsilon}}\left(y_{j}+\epsilon, y_{j}-\epsilon\right)} \Omega e^{i S / \hbar}=1+\alpha_{j} .
\end{gathered}
$$

Заметим, что эти величины совпадают с хорошо известными коэффициентами отражения и поглощения от ступеньки.

ОПРЕДЕЛЕНиЕ 7. Пути в ступенчатом потенциале определяются следующим образом:

$$
\begin{aligned}
\widetilde{\operatorname{Paths}}\left(x^{\prime}, x^{\prime \prime}\right) & =\left\{\left(j_{1}, \ldots, j_{N}\right) \mid x^{\prime} \in \Delta_{j^{\prime}} \Rightarrow j_{1} \in\left\{j^{\prime}-1, j^{\prime}\right\},\right. \\
j_{k+1} & \left.\in\left\{j_{k}-1, j_{k}+1\right\}(1 \leqslant k \leqslant N), x^{\prime \prime} \in \Delta_{j^{\prime \prime}} \Rightarrow j_{N} \in\left\{j^{\prime \prime}-1, j^{\prime \prime}\right\}\right\} .
\end{aligned}
$$

ЗАмЕчаниЕ 4. Посредством $N(P)$ мы будем обозначать число $N$ для элемента $P \in \widetilde{\text { Paths, }}$ фигурирующее в определении 7. Иногда будем использовать индексы

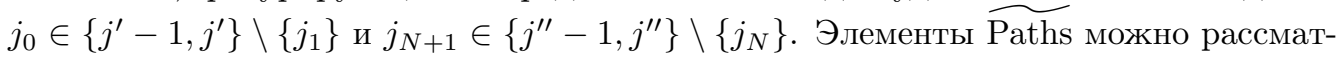
ривать как случайные блуждания между точками $y_{j}$. 
ОПРЕдЕЛЕниЕ 8 . Пусть $P=\left(j_{1}, \ldots, j_{N}\right) \in \widehat{\operatorname{Paths}}\left(x^{\prime}, x^{\prime \prime}\right)$. Тогда коэффициенты отражения и поглощения можно записать как

$$
A_{k}[P]=\left\{\begin{array}{lll}
1-\alpha_{j_{k}}, & j_{k-1}=j_{k}-1, & j_{k+1}=j_{k}+1, \\
1+\alpha_{j_{k}}, & j_{k-1}=j_{k}+1, & j_{k+1}=j_{k}-1, \\
-\alpha_{j_{k}}, & j_{k-1}=j_{k}-1, & j_{k+1}=j_{k}-1, \\
\alpha_{j_{k}}, & j_{k-1}=j_{k}+1, & j_{k+1}=j_{k}+1,
\end{array} \quad 1 \leqslant k \leqslant N .\right.
$$

ОПРЕДЕЛЕНИЕ 9. Пусть $P=\left(j_{1}, \ldots, j_{N}\right) \in \widetilde{\operatorname{Paths}}\left(x^{\prime}, x^{\prime \prime}\right)$ и $x^{\prime} \in \Delta_{j^{\prime}}, x^{\prime \prime} \in \Delta_{j^{\prime \prime}}$, $\left[y_{j_{k}}, y_{j_{k+1}}\right]=\Delta_{l_{k}}$. Тогда действие имеет вид

$$
S[P]=p_{j^{\prime}}\left|x^{\prime}-y_{j_{1}}\right|+\sum_{k=1}^{N-1} p_{l_{k}} a_{l_{k}}+p_{j^{\prime \prime}}\left|y_{j_{N}}-x^{\prime \prime}\right|
$$

Учитывая все вышесказанное, несложно прийти к следующей теореме.

Теорема 2. В случае ступенчатого потенииала $U(x)$

$$
\left\langle x^{\prime \prime}\left|\frac{1}{H-E}\right| x^{\prime}\right\rangle=\frac{1}{\sqrt{p\left(x^{\prime}\right) p\left(x^{\prime \prime}\right)}} \sum_{P \in \operatorname{Paths}\left(x^{\prime}, x^{\prime \prime}\right)} \prod_{k=1}^{N(P)} A_{k}[P] e^{i S[P]} .
$$

Заметим, что произвольный потенциал можно представить как предел ступенчатого потенциала и получить (22) из (42).

Можно рассмотреть простейший случай, когда имеется лишь один интервал постоянного потенциала, ограниченный бесконечно высокими стенками (т.е. частицу в ящике). Тогда наше представление имеет смысл пересуммирования по Пуассону результата, полученного с помощью уравнения Шредингера. Таким образом, общий случай можно рассматривать как обобщение формулы пересуммирования Пуассона.

\section{5. КВАЗИКЛАССИЧЕСКИЙ ПРЕДЕЛ}

Мы можем восстановить амплитуду перехода $K_{T}\left(x^{\prime}, x^{\prime \prime}\right)$ (для которой пишется представление в виде обычного функционального интеграла) во временно́м представлении следующим простым образом (это обратное преобразование Лапласа):

$$
\begin{aligned}
K_{T}\left(x^{\prime}, x^{\prime \prime}\right)= & \oint_{\substack{\text { eigenvalues } \\
\text { of } H}} \frac{d E}{2 \pi i \hbar} K_{E}\left(x^{\prime}, x^{\prime \prime}\right) e^{-E T / \hbar}= \\
=- & \oint \frac{d E}{2 \pi i \hbar} \frac{1}{\sqrt{p\left(x^{\prime}\right) p\left(x^{\prime \prime}\right)}} \sum_{N=0}^{\infty} \int_{\operatorname{Paths}\left(x^{\prime}, x^{\prime \prime}\right)} \prod_{k=1}^{N} d x_{k} \frac{p^{\prime}\left(x_{k}\right)}{2 p\left(x_{k}\right)} \times \\
& \times(-1)^{\rightleftarrows} \exp \left\{\frac{i}{\hbar} \int_{P} p(\xi) d \xi-\frac{1}{\hbar} E T\right\}
\end{aligned}
$$

Рассмотрим теперь квазиклассический предел этой формулы для амплитуды перехода $K_{T}\left(x^{\prime}, x^{\prime \prime}\right)$. Для каждой траектории $P \in \operatorname{Paths}\left(x^{\prime}, x^{\prime \prime}\right)$ можно взять интеграл 
от соответствующего члена по $E$ методом перевала асимптотически при $\hbar \rightarrow 0$ :

$$
\begin{gathered}
-\oint \frac{d E}{2 \pi i \hbar} \frac{1}{\sqrt{p\left(x^{\prime}, E\right) p\left(x^{\prime \prime}, E\right)}} A[P](E) e^{i S[P](E) / \hbar} e^{-E T / \hbar}= \\
=\sqrt{\frac{1}{2 \pi \hbar\left(\partial T_{P} / \partial E\right)} \frac{A[P]}{\sqrt{p\left(x^{\prime}\right) p\left(x^{\prime \prime}\right)}} e^{-(-i S[P](E)+E T) / \hbar} \times} \\
\times\left.\left(1+c_{1} \hbar+c_{2} \hbar^{2}+\cdots\right)\right|_{i \frac{\partial}{\partial E}} S[P](E) \equiv T[P](E)=T
\end{gathered}
$$

где $A[P]=\prod_{k=1}^{N}(-1){ }^{k} p^{\prime}\left(x_{k}\right) /\left(2 p\left(x_{k}\right)\right)$. Обозначим $E_{\mathrm{Eucl}} \equiv-E, U_{\mathrm{Eucl}}(x) \equiv-U(x)$, $p_{\text {Eucl }}(x) \equiv \sqrt{2\left(E_{\text {Eucl }}-U_{\text {Eucl }}(x)\right)}=\sqrt{2(U(x)-E)}$. Можно показать, что точка перевала всегда такова, что $E$ вещественно и $E_{\mathrm{Eucl}}>\max _{x \in P} U_{\mathrm{Eucl}}(x) \Rightarrow p_{\mathrm{Eucl}}(x)>0$ на $P$. Следовательно, в показателе экспоненты мы получим полное евклидово действие $^{5)}$

$$
S_{\mathrm{Eucl}}^{\mathrm{full}}=-i S[P](E)+E T=\int_{P} p_{\mathrm{Eucl}}(\xi) d \xi-E_{\mathrm{Eucl}} T \text {. }
$$

Уравнение для седловой точки выглядит как

$$
T=i \frac{\partial S[P](E)}{\partial E}=\int_{P} \frac{d \xi}{p_{\text {Eucl }}(\xi)} .
$$

Его правая часть есть классическая формула для вычисления времени на данной траектории, т.е. из этого уравнения следует, что энергия $E$ такова, что время движения вдоль пути $P$ равняется $T$. О таком движении можно думать как о “локально классическом", потому что оно классическое везде, кроме возможных неклассических точек отражения $x_{k}$, где потенциал $U_{\text {Eucl }}(x)$ меньше, чем $E_{\text {Eucl }}$. С точки зрения функционального интеграла вместо обычного разложения около настоящей классической траектории мы производим разложение около “локально классических" траекторий, множество которых более богато ${ }^{6)}$. Бесконечный ряд по $\hbar$ в формуле (44) соответствует флуктуациям около этих траекторий. Каждый член может быть записан в терминах производных $S[P](E)$ и $A[P](E)$ по $E$.

Рассмотрим амплитуду перехода в случае, когда чисто классическая траектория не имеет точек поворота. Тогда в ряде (43) член с $N$ точками отражения вносит вклад только в $O\left(\hbar^{[(N+1) / 2]}\right)$ - часть асимптотического разложения $K\left(T, x^{\prime}, x^{\prime \prime}\right)$ в пределе $\hbar \rightarrow 0$. Эти члены даются траекториями с отражениями, расположенными в малой окрестности $(\sim \hbar)$ классической траектории.

Главный порядок в разложении члена с $N=0$ дает правильный однопетлевой ответ. Это следует из того, что

$$
\frac{p\left(x^{\prime \prime}\right) p\left(x^{\prime}\right)}{m} \frac{\partial T_{P}}{\partial E}=\operatorname{Det}_{t_{1}, t_{2}} \frac{\delta^{2} S_{P}^{\text {full }}[x]}{\delta x\left(t_{1}\right) \delta x\left(t_{2}\right)} .
$$

\footnotetext{
5) Переход от укороченного действия к полному и обратно есть преобразование Лежандра: $S^{\text {short }}(E)-E T=S^{\text {full }}(T), T=\partial S^{\text {short }} / \partial E, E=-\partial S^{\text {full }} / \partial T$.

6) Конечно, после интегрирования по точкам отражения $x_{k}$ в пределе $\hbar \rightarrow 0$ в непрерывном потенциале останутся лишь истинные классические траектории, как описано ниже.
} 
Чтобы доказать это, следует стандартным образом представить определитель как решение дифференциального уравнения. В случае, когда чисто классическая траектория имеет точки поворота, это утверждение следует несколько изменить (см. ниже); грубо говоря, поправки даются траекториями с отражениями, которые не лежат в очень маленькой окрестности классических точек поворота.

Известно, что не существует настоящего классического многоинстантонного решения в системе с потенциалом с двумя ямами. Но оказывается, что такие конфигурации близки к полученным “локально классическим” траекториям. Объяснение можно найти в следующем разделе.

Вместо взятия интеграла по $E$, как ранее, можно сначала произвести квазиклассическое интегрирование по точкам поворота $x_{k}$. Прежде всего, мы должны деформировать контур интегрирования по $E$ так, чтобы он лежал вплотную к вещественной оси (где расположены интересующие нас полюсы): $E=\mathcal{E} \pm i \epsilon$, где $\mathcal{E}$ вещественно. Основной вклад тогда будет даваться конфигурациями, в которых все $x_{k}$ лежат в окрестностях точек $\mathcal{E}=U(x)$. Можно просуммировать отражения в одной такой классической точке поворота ${ }^{7)}$ и получить квазиклассические правила суммирования для $K_{E}$ или $Z_{E}$ : суммировать нужно по всем траекториям с отражениями только в корнях ${ }^{8)}$ уравнения $\mathcal{E}=U(x)$ с множителями $+i$ или $-i$ для отражений в случаях, когда траектория выходит из области, где потенциал соответственно больше или меньше $\mathcal{E}$. В случае одноямного потенциала (два корня) это дает нам (в результате простого суммирования геометрической прогрессии) выражение типа $(\cdots) /(1+\exp \{i \oint p d x / \hbar\})$, структура полюсов которого дает правило квантования Бора-Зоммерфельда (посредством $(\cdots)$ здесь и ниже мы обозначаем некое аналитическое по $E$ выражение).

\section{6. ИНСТАНТОНЫ}

Рассмотрим теперь случай потенциала с двойной ямой. Пусть энергия такова, что $\mathcal{E}=U(x)$ имеет четыре корня (обозначим их слева направо $x_{1}, x_{2}, x_{3}, x_{4}$ ). Пусть

$$
S=\int_{x_{1}}^{x_{2}} p d x=\int_{x_{3}}^{x_{4}} p d x, \quad R=\int_{x_{2}}^{x_{3}}|p| d x .
$$

Тогда описанное суммирование (здесь снова возникает просто геометрическая прогрессия, с учетом уже посчитанной выше суммы для траекторий между парами $\left(x_{1}, x_{2}\right)$ и $\left.\left(x_{3}, x_{4}\right)\right)$ даст

$$
Z_{E} \sim \frac{(\cdots)}{e^{-2 R / \hbar}+\left(e^{2 i S / \hbar}+1\right)^{2}} .
$$

Для осциллятора $S=2 \pi i E /(\hbar \omega)$. Пусть $E=E^{0}+\Delta E, E^{0}=\hbar \omega(n+1 / 2), \Delta E \ll E$. Тогда полученное уравнение дает

$$
\left(\frac{\Delta E 2 \pi}{\hbar \omega}\right)^{2}=e^{-2 R / \hbar} \Rightarrow \Delta E= \pm \frac{\hbar \omega}{2 \pi} e^{-R / \hbar}
$$

\footnotetext{
7) Практически это исследование соответствующих уравнений в окрестностях точек поворота.

8) Чтобы получить выражение с аналитически правильным по $E$ поведением, нужно рассматривать также комплексные корни.
} 
что представляет собой в точности известный результат (см., например, [2]). Член $e^{-2 R / \hbar}$ в знаменателе (46), который сдвигает полюс, берется из геометрической прогрессии, $N$-й член которой содержит $e^{-2 N R / \hbar}$. Он соответствует пути, проходящему $2 N$ раз от одной вершины к другой, и, таким образом, содержит $2 N$ инстантонов.

Известно вычисление расщепления энергетического уровня с помощью инстантонов (см., например, [3] или [4]), которое предполагает, что основной вклад в функциональный интеграл дают конфигурации с $N$ кинками с произвольными положениями. Рассмотрим классические траектории в перевернутом потенциале. Классическая траектория, которая соединяет одну вершину с другой, содержит лишь один инстантон при любом конечном $T$. Если же мы рассмотрим классические траектории при энергии меньшей, чем максимум потенциала, то возможны решения с $N$ инстантонами. Но решение, очевидно, должно быть периодично, и, следовательно, расстояния между центрами всех этих инстантонов одинаковы.

Покажем теперь, как можно объяснить тот факт, что при очень большом $T$ основной вклад действительно дают траектории, в которых частица произвольное время находится в вершине потенциала. Когда $\hbar \rightarrow 0$ в формуле (43), основной вклад вносят пути с поворотами в точках $E=U(x)$. Как уже было отмечено, каждый такой поворот дается суммой по произвольным комбинациям с отражениями, сосредоточенными в малой окрестности корня. При этом можно оценить, какое в среднем дополнительное время частица из-за этого проводит в окрестности этой точки (в предположении, что оно мало по сравнению с $T): \Delta T \sim\left[\hbar m /\left(U^{\prime}\right)^{2}\right]^{1 / 3} \propto \hbar^{1 / 3} e^{\omega T /(3 N)}$ для $N$ инстантонов. Когда $\hbar \rightarrow 0$ (а $T$ фиксировано), конечно, $\Delta T \rightarrow 0$. Но если $\hbar$, хоть и очень мало, но фиксировано ${ }^{9)}$, отношение $\Delta T / T$ растет очень быстро при $T \rightarrow \infty$. Полученная оценка тогда становится неверной, и $\Delta T / T \rightarrow 1$ (выходит на насыщение). Таким образом, квантовые отражения в окрестности вершины потенциала как раз и приводят к тому, что частица может стоять произвольное время в вершине потенциала, когда энергия близка к нулю (время стремится к бесконечности).

В приложении Г показано, как стандартное вычисление расщепления уровня энергии может быть воспроизведено через полученное представление для амплитуды перехода. Проще говоря, геометрическая прогрессия, дающая сдвиг полюса в энергетическом представлении, переходит в ряд для экспоненты во временно́м представлении.

Заметим, что одной из главных особенностей преобразования Лапласа является переход свертки по времени в умножение. Поэтому функциональный интеграл как сумма по различным последовательностям событий за определенное время переходит в сумму по последовательностям независимых событий, т.е. таких, что в каждой точке своего пути частица "не помнит" предысторию, и веса, соответствующие путям, имеют мультипликативную структуру (с точностью до краевых множителей):

$$
A\left[P_{1} \sqcup P_{2}\right] e^{i S\left[P_{1} \sqcup P_{2}\right]}=A\left[P_{1}\right] e^{i S\left[P_{1}\right]} \cdot A\left[P_{2}\right] e^{i S\left[P_{2}\right]} .
$$

9) Формально достаточно, чтобы $\hbar \rightarrow 0$ как степень $T$. 
Такая форма дает также достаточно простой способ описания систем, составленных из некоторых более простых подсистем. В случае потенциала с двойной ямой это две квадратичных ямы и промежуточная область, в случае ступенчатого потенциала сами ступеньки.

\section{7. НЕПОСРЕДСТВЕННОЕ ОБОБЩЕНИЕ ФОРМУЛ (9) И (22) НА D-МЕРНУЮ КВАНТОВУЮ МЕХАНИКУ}

Представление (9) формулы (22) подсказывает один из возможных способов обобщения на случай $D$-мерной квантовой механики. Чтобы получить (9) в размерности $D$, мы должны произвести расширение на алгебру Клиффорда. А именно, ничего не изменится в доказательстве формулы (9), если сделать следующую замену:

$$
\partial \rightarrow \hat{\partial} \equiv \gamma_{a} \partial_{a}, \quad p^{\prime} \rightarrow(\hat{\partial} p), \quad\left\{\gamma_{a}, \gamma_{b}\right\}=2 \delta_{a b}, \quad a, b=1, \ldots, D .
$$

В таком расширении нет ничего плохого, так как итоговый ответ будет в $\mathbb{C}$-компоненте. В матричном представлении мы можем взять $2^{-[D / 2]} \operatorname{Tr}_{\gamma}$ от каждого члена, чтобы написать формулу просто в $\mathbb{C}$ :

$$
\begin{gathered}
\frac{2 i}{p(x)^{2}+\Delta}=2^{-[D / 2]} \operatorname{Tr}_{\gamma}\left[\frac{1}{\sqrt{p}} \frac{i}{p+i \hat{\partial}} \frac{1}{\sqrt{p}}+\frac{1}{\sqrt{p}} \frac{i}{p-i \hat{\partial}} \frac{1}{\sqrt{p}}+\right. \\
\left.+\frac{1}{\sqrt{p}} \frac{i}{p+i \hat{\partial}} \frac{(\hat{\partial} p)}{2 p} \frac{i}{p-i \hat{\partial}} \frac{1}{\sqrt{p}}+\cdots\right] .
\end{gathered}
$$

Ядра операторов $i /(p \pm i \hat{\partial})$ можно представить следующим образом. Известно следующее представление для пропагатора Дирака [5], [6]:

$$
\begin{aligned}
G_{m}\left(x^{\prime \prime}, x^{\prime}\right) & \equiv\left\langle x^{\prime \prime}\left|\frac{1}{m+\hat{\partial}}\right| x^{\prime}\right\rangle= \\
& =\sum_{P \in\left\{\text { paths } x^{\prime} \rightarrow x^{\prime \prime}\right\}} e^{-m L_{P}} \cdot \mathcal{P} \exp \left\{\int_{P} d s \frac{1}{4} \omega_{a b}(s)\left[\gamma_{a}, \gamma_{b}\right]\right\}
\end{aligned}
$$

где $L_{P}=\int_{P} d s$ - длина пути $P, s$ - естественный параметр вдоль кривой $P$ и $\omega_{a b}=$ $\dot{x}_{[a} \ddot{x}_{b]}-$ угловая скорость касательного вектора. Второй множитель в формуле $(50)-$ так называемый спиновый фактор, а $\mathcal{P}$ - оператор пространственного упорядочения вдоль кривой $P$.

Мы можем обобщить формулу (50) для наших целей следующим образом:

$$
\begin{aligned}
G_{[p]}\left(x^{\prime \prime}, x^{\prime}\right) & \equiv\left\langle x^{\prime \prime}\left|\frac{i}{p+i \hat{\partial}}\right| x^{\prime}\right\rangle= \\
& =\sum_{P \in\left\{\text { paths } x^{\prime} \rightarrow x^{\prime \prime}\right\}} \exp \left\{i \int_{P} d s p(x(s))\right\} \cdot \mathcal{P} \exp \left\{\int_{P} d s \frac{1}{4} \omega_{a b}(s)\left[\gamma_{a}, \gamma_{b}\right]\right\} .
\end{aligned}
$$


Эту формулу можно доказать, используя (50) и варьируя $p(x) \rightarrow p(x)+\epsilon \phi(x)$ в правой части (51):

$$
\begin{aligned}
\int d y \phi(y) \frac{\delta}{\delta p(y)} G_{[p]} & =i G_{[p]} \phi G_{[p]} \Leftrightarrow \frac{\delta}{\delta p(y)} G_{[p]}^{-1}= \\
& =-i \delta(x-y) \Leftrightarrow G_{[p]}=\frac{1}{\text { const }-i p(x)},
\end{aligned}
$$

и тогда, если выражение (51) верно для $p(x)=i m$ (оно становится эквивалентно $(50))$, то оно верно и для произвольного $p(x)$.

Теперь мы можем использовать формулу (49) (наше обобщение разложения (8)), чтобы написать обобщение $(22)$ для $D>1$ :

$$
\begin{aligned}
&\left\langle x^{\prime}\left|\frac{\hbar}{H-E}\right| x^{\prime \prime}\right\rangle= \\
&=2^{-[D / 2]} \oint \frac{1}{\sqrt{p\left(x^{\prime}\right) p\left(x^{\prime \prime}\right)}} \sum_{N=0}^{\infty} \sum_{r=0}^{1}(-1)^{[(N+r) / 2]} \int \prod_{k=1}^{N} d x_{k} \operatorname{Tr}_{\gamma} \mathcal{P} \frac{(\hat{\partial} p)\left(x_{k}\right)}{2 p\left(x_{k}\right)} \times \\
& \quad \times \prod_{j=0}^{N} \sum_{P_{j} \in\left\{\text { paths } x_{j} \rightarrow x_{j+1}\right\}} \exp \left\{\frac{1}{\hbar} S\left[P_{j}\right]+\frac{(-1)^{r+j}}{4} \int_{P_{j}} d s \omega_{a b}(s)\left[\gamma_{a}, \gamma_{b}\right]\right\}
\end{aligned}
$$

где $S[P]=i \int_{P} p(x(s)) d s$ и мы опять отождествили $x_{0} \equiv x^{\prime}, x_{N+1} \equiv x^{\prime \prime}$.

Таким образом, мы получили представление амплитуды перехода через сумму по траекториям в $x$-пространстве (это в самом деле геометрические траектории, а не функции $x(t)$, как в стандартном подходе). И опять веса для траекторий имеют мультипликативную структуру (46). Следствия этого (независимость от предыстории и т.д.) уже обсуждались.

Функциональный интеграл в формулах (50) и (51) уже не очень хорошо определен, но имеет следующую особенность: пути, которые вносят вклад в интеграл, имеют хаусдорфову размерность 1 (в отличие от функционального интеграла для бозонного пропагатора, где траектории имеют размерность 2). Это происходит из-за наличия спинового фактора, который подавляет траектории с большой кривизной, и поэтому следует учитывать только гладкие траектории. В случае $D=1^{10)}$, рассмотренном выше, траектории даже не могут менять направление.

Мы можем рассматривать как (53), так и (22) как разложение по $N$, т.е. количеству точек разрыва касательной к траектории как кривой в $x$-пространстве.

\section{8. ЗАКЛЮЧЕНИЕ}

Отметим несколько более или менее интересных (с субъективной точки зрения автора) направлений дальнейших исследований.

1. Интересно было бы найти обобщение (лучше, чем в разделе 7 ) на $D$-мерную квантовую механику, т.е. повысить размерность таргет-пространства.

10) Формулы, приведенные в этом разделе, однако, не могут быть применены в случае $D=1$, они написаны для $D>1$. 
2. Обобщение на квантовую теорию поля, т.е. повышение размерности мирового пространства, также кажется интересным.

Отрицательные результаты также могут представлять интерес. Можно попытаться сформулировать теоремы, запрещающие обобщения на высшие измерения.

3. Хотелось бы найти явную связь представления (21) с обычным функциональным интегралом. Определим функциональное пространство $\Gamma\left(x^{\prime}, x^{\prime \prime}\right)=\{(T>0$, $\left.\left.x(\cdot) \in C^{1}([0, T], V)\right) \mid x(0)=x^{\prime}, x(T)=x^{\prime \prime}\right\}$ (время движения не фиксировано). Тогда можно ввести проекцию $\pi: \Gamma\left(x^{\prime}, x^{\prime \prime}\right) \rightarrow \operatorname{Paths}\left(x^{\prime}, x^{\prime \prime}\right)$ - "забывание" зависимости $x$ от $t$, и сохранение только $x$-позиции локальных максимов и минимумов ${ }^{11)}-x_{k}$. Тогда было бы интересно получить соотношение типа (возможно, с некоторой корректировкой)

$$
\frac{1}{\sqrt{p\left(x^{\prime}\right) p\left(x^{\prime \prime}\right)}} \int_{\Lambda} \Omega e^{i S}=\int_{\pi^{-1} \Lambda} d T \mathcal{D} x e^{-S^{\text {full }}[x]+E T}
$$

для всех $\Lambda \subset \operatorname{Paths}\left(x^{\prime}, x^{\prime \prime}\right)$; т.е. меру $\mu: \mu(\Lambda)=\int_{\Lambda} \Omega e^{i S}$, которую мы строим на $\operatorname{Paths}\left(x^{\prime}, x^{\prime \prime}\right)$, можно рассматривать как прямой образ $\mu=\pi_{*} \tilde{\mu}$ гипотетической меры $\tilde{\mu}$ на $\Gamma\left(x^{\prime}, x^{\prime \prime}\right)$ при действии проекции $\Gamma\left(x^{\prime}, x^{\prime \prime}\right) \stackrel{\pi}{\rightarrow} \operatorname{Paths}\left(x^{\prime}, x^{\prime \prime}\right)$. Мотивацией для такого соотношения является локальность функционалов на траекториях в обеих частях равенства. Когда мы изменяем потенциал $U(x)$ в окрестности некоторой точки, изменится только часть суммы, содержащая траектории, проходящие через эту точку. В данной работе мы доказали это равенство для $\Lambda=\operatorname{Paths}\left(x^{\prime}, x^{\prime \prime}\right)$.

4. Интересно было бы найти связи с теорией случайных блужданий.

\section{ПРИЛОЖЕНИЕ А}

\section{Пертурбативный тест формулы (9)}

Покажем, как формула (9) согласуется с пертурбативным подходом к амплитуде перехода в первом порядке. А именно, рассмотрим систему (положим $\hbar=1$ и $m=1)$ с $H=-(1 / 2) \partial^{2}+\lambda V(x) \equiv H_{0}+\lambda V(x)$ и $\lambda \rightarrow 0$. Для оператора эволюции $K(T)=e^{-H T}$ имеем следующее стандартное пертурбативное разложение по $\lambda$ :

$$
K(T)=K_{0}(T)-\lambda \int_{0}^{T} d \tau K_{0}(T-\tau) V(x) K_{0}(\tau)+\cdots,
$$

где $K_{0}=e^{-H_{0} T}$. В энергетическом представлении этот ряд - просто геометрическая прогрессия:

$$
K_{0}(E)=\frac{-1}{E-\lambda V+\partial^{2} / 2}=-\left(\frac{1}{E+\partial^{2} / 2}+\frac{1}{E+\partial^{2} / 2} \lambda V \frac{1}{E+\partial^{2} / 2}+\cdots\right) .
$$

Покажем, что в первом порядке по $\lambda$ это совпадает с (9). С такой точностью мы имеем

$$
\begin{gathered}
p^{2}=p_{0}^{2}\left(1-\frac{\lambda V}{p_{0}^{2}}\right), \quad \frac{p^{\prime}}{2 p}=-\frac{\lambda V^{\prime}}{2 p_{0}^{2}}, \quad p_{0} \equiv \sqrt{2 E} \\
\frac{1}{p \pm i \partial}=\frac{1}{p_{0} \pm i \partial}+\frac{1}{p_{0} \pm i \partial} \frac{\lambda V}{p_{0}^{2}} \frac{1}{p_{0} \pm i \partial}
\end{gathered}
$$

\footnotetext{
11) В областях с постоянным потенциалом их также следует забыть.
} 
В таком порядке по $\lambda$ нам нужны, очевидно, только члены из (8), (9) с не более чем одним отражением ${ }^{12)}$. В нулевом порядке согласование тривиально:

$$
\frac{2 p_{0}}{p_{0}^{2}+\partial^{2}}=\frac{1}{p_{0}+i \partial}+\frac{1}{p_{0}-i \partial} .
$$

После выделения членов, дающих первый порядок, остается проверить, что

$$
\begin{aligned}
4 p_{0}^{2} & \frac{1}{p_{0}^{2}+\partial^{2}} V \frac{1}{p_{0}^{2}+\partial^{2}}=i\left\{\frac{1}{p_{0}-i \partial} \frac{V^{\prime}}{2 p_{0}^{2}} \frac{1}{p_{0}+i \partial}-\frac{1}{p_{0}+i \partial} \frac{V^{\prime}}{2 p_{0}^{2}} \frac{1}{p_{0}-i \partial}\right\}+ \\
& +\frac{V}{2 p_{0}^{2}} \frac{2 p_{0}}{p_{0}^{2}+\partial^{2}}+\frac{2 p_{0}}{p_{0}^{2}+\partial^{2}} \frac{V^{\prime}}{2 p_{0}^{2}}+\frac{1}{p_{0}+i \partial} \frac{V}{p_{0}^{2}} \frac{1}{p_{0}+i \partial}+\frac{1}{p_{0}-i \partial} \frac{V}{p_{0}^{2}} \frac{1}{p_{0}-i \partial} .
\end{aligned}
$$

Мы можем умножить это соотношение на $\left(p_{0}^{2}+\partial^{2}\right)$ слева и справа. После алгебраических преобразований получаем

$$
4 V p_{0}^{2}=-\left[\partial, V^{\prime}\right]+4 V p_{0}^{2}+\left\{\partial^{2}, V\right\}-2 \partial V \partial .
$$

В правильности этого тождества легко убедиться.

\section{Доказательство формулы (35)}

ПРИЛОЖКЕНИЕ Б

Формула

$$
\int_{\operatorname{Paths}(x, x)} \Omega e^{i S}=\frac{J_{\mathrm{R}}\left(x^{\prime}, x^{\prime \prime}\right)\left(1+I_{\mathrm{L}}\left(x^{\prime}\right)\right)}{1-I_{\mathrm{L}}\left(x^{\prime}\right) I_{\mathrm{R}}\left(x^{\prime}\right)}
$$

может быть получена аналогично (30) путем суммирования двух геометрических прогрессий в точке $x^{\prime}$.

Заметим, что если положить $x^{\prime}=x^{\prime \prime}=x_{0}$ (используя (34)) и проинтегрировать по $x_{0}$, мы получим формулу (30).

Достаточно показать, что

$$
\frac{1}{\sqrt{p\left(x^{\prime}\right) p\left(x^{\prime \prime}\right)}} \frac{J_{\mathrm{R}}\left(x^{\prime}, x^{\prime \prime}\right)\left(1+I_{\mathrm{L}}\left(x^{\prime}\right)\right)}{1-I_{\mathrm{L}}\left(x^{\prime}\right) I_{\mathrm{R}}\left(x^{\prime}\right)}=-2 \frac{\psi_{\mathrm{L}}\left(x^{\prime}\right) \psi_{\mathrm{R}}\left(x^{\prime \prime}\right)}{W},
$$

чтобы получить формулу (35). Используя выражение (28), приходим к тому, что это равносильно

$$
\frac{i}{\sqrt{p\left(x^{\prime}\right) p\left(x^{\prime \prime}\right)}} J_{\mathrm{R}}\left(x^{\prime}, x^{\prime \prime}\right)\left(\psi_{\mathrm{R}}^{\prime}\left(x^{\prime}\right)+i p\left(x^{\prime}\right) \psi_{\mathrm{R}}\left(x^{\prime}\right)\right)=-2 \psi_{\mathrm{R}}\left(x^{\prime \prime}\right) .
$$

При $x^{\prime}=x^{\prime \prime}$ последнее равенство, очевидно, выполняется (подставляем (34) и (28)). Поэтому остается лишь доказать, что величина

$$
A(x) \equiv \frac{J_{\mathrm{R}}\left(x, x^{\prime \prime}\right)\left(\psi_{\mathrm{R}}^{\prime}(x)+i p(x) \psi_{\mathrm{R}}(x)\right)}{\sqrt{p(x)}}
$$

12)Это общая особенность: в порядок $O\left(\lambda^{N}\right)$ вносят вклад только траектории с не более чем $N$ отражениями. 
не зависит от $x$. Для этого, используя (33), вычислим ее логарифмическую производную:

$$
(\ln A)^{\prime}=-i p-\frac{p^{\prime}}{2 p} I_{\mathrm{R}}-\frac{p^{\prime}}{2 p}+\frac{\psi_{\mathrm{R}}^{\prime \prime}+i p \psi_{\mathrm{R}}^{\prime}+p^{\prime} \psi_{\mathrm{R}}}{\psi_{\mathrm{R}}^{\prime}+i p \psi_{\mathrm{R}}} .
$$

Подставляя сюда $I_{\mathrm{R}}$ из $(28)$ и $\psi_{\mathrm{R}}^{\prime \prime}=-p^{2} \psi_{\mathrm{R}}$ и производя чисто алгебраические упрощения, убеждаемся, что действительно $(\ln A)^{\prime}=0$.

\section{Доказательство формулы (37)}

ПРИЛОЖКЕНИЕ В

Докажем, что

$$
\int_{\operatorname{Paths}_{[a, b]}(a, a)} \Omega=\sum_{n=0}^{\infty}(-1)^{n} \int_{\left\{T \geqslant t_{2 i \pm 1} \geqslant t_{2 i} \geqslant 0\right\}} \prod_{k=1}^{2 n+1} d t_{k}=\operatorname{th} T=\frac{p(b)-p(a)}{p(b)+p(a)}
$$

где мы использовали переменные $t=\ln [p(x) / p(a)] / 2, T=\ln [p(b) / p(a)] / 2$. Эту формулу можно аналогичным образом представить в виде (по внутренним точкам производится интегрирование от 0 до $T$ )

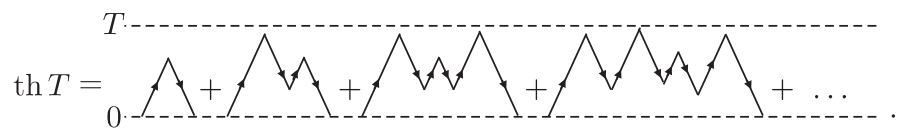

Тогда она может быть записана как

$$
\text { th } T=G(0,0)
$$

где $G\left(t_{2}, t_{1}\right)$ - ядро оператора $G$, являющегося следующей суммой:

$$
G=K-K^{2}+K^{3}-K^{4}+\cdots=\frac{K}{1+K} .
$$

Оператор $K$ действует на функции следующим образом:

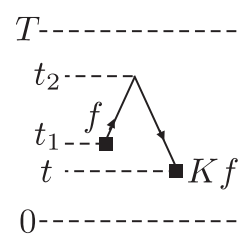

$$
(K f)(t)=\int_{t}^{T} d t_{2} \int_{0}^{t_{2}} d t_{1} f\left(t_{1}\right)
$$

Несложно понять, что $(K f)(T)=0,(K f)^{\prime}(0)=0,(K f)^{\prime \prime}(t)=-f(t)$. Но тогда $K=-\partial^{-2}$ на пространстве функций $\{\phi\}$ на отрезке $[0, T]$ с граничными условиями $\phi(T)=0$ и $\phi^{\prime}(0)=0$. Поэтому оператор $G$ на таком пространстве равен

$$
G=\frac{1}{-\partial^{2}+1},
$$


и его ядро можно записать стандартным образом через функции, удовлетворяющие уравнению $\left(\partial_{t}^{2}-1\right) \phi(t)=0$ : функцию $\operatorname{sh}(T-t)$, удовлетворяющую первому граничному условию, и функцию $\operatorname{ch} t$, удовлетворяющую второму, а также их вронскиан $W=-\operatorname{ch} T:$

$$
\begin{aligned}
G\left(t_{2}, t_{1}\right) & =\frac{\operatorname{ch} t_{<} \operatorname{sh}\left(T-t_{>}\right)}{\operatorname{ch} T}, \\
t_{>}=\max \left\{t_{2}, t_{1}\right\}, \quad t_{<} & =\min \left\{t_{2}, t_{1}\right\} \Rightarrow G(0,0)=\operatorname{th} T,
\end{aligned}
$$

что и требовалось доказать.

Заметим, что при попытке доказывать равенство (П.12) напрямую возникает следующее нетривиальное комбинаторное утверждение: объем части единичного $(2 n-1)$-мерного куба, ограниченной неравенствами $\left\{1 \geqslant x_{2 i \pm 1} \geqslant x_{2 i} \geqslant 0\right\}$, равен $B_{2 n} \cdot 2^{2 n}\left(2^{2 n}-1\right) /(2 n)$ !, где $B_{k}$ - числа Бернулли.

\section{ПРИЛОЖКНИЕ Г}

\section{Вычисление расщепления уровня энергии с помощью представления (22)}

Рассмотрим амплитуду перехода в энергетическом представлении в двухъямном потенциале (который переворачивается в евклидовом случае) от одной вершины к другой. Пусть вершины расположены в точках $-a$ и $a$. Нас интересует случай, когда $T \rightarrow \infty$, т.е. самый левый полюс по $E$. Опираясь на вышесказанное, в формуле (43) мы пренебрегаем траекториями с отражениями вне интервалов $[-a-b,-a+b]$ и $[a-b, a+b]$. Введем основные составляющие нашей суммы.

1. "Инстантонный оператор" с ядром:

$$
I\left(x^{\prime \prime}, x^{\prime}\right)=\exp \left(\frac{i}{\hbar}(-1)^{\theta\left(x^{\prime}-x^{\prime \prime}\right)} \int_{x^{\prime}}^{x^{\prime \prime}} p(\xi) d \xi\right)
$$

где $x_{1}$ и $x_{2}$ лежат в разных отрезках $[-a-b,-a+b]$ и $[a-b, a+b]$. Эти две окрестности на самом деле симметричны, и мы можем рассматривать для удобства лишь одну копию (назовем ее $\Delta$ ) и помнить только, что полное число инстантонов должно быть нечетным.

2. Сумма по траекториям, лежащим в $\Delta$ между двумя инстантонами:

$$
Y\left(x^{\prime \prime}, x^{\prime}\right)=\int_{\operatorname{Paths}_{\Delta}\left(x^{\prime}, x^{\prime \prime}\right)} \Omega e^{i S} .
$$

3. Начальная и конечная части:

$$
Y_{\text {in }}\left(x^{\prime \prime}\right)=\int_{\operatorname{Paths}_{\Delta}\left(-a, x^{\prime \prime}\right)} \Omega e^{i S}, \quad Y_{\text {out }}\left(x^{\prime}\right)=\int_{\operatorname{Paths}_{\Delta}\left(x^{\prime}, a\right)} \Omega e^{i S}:
$$




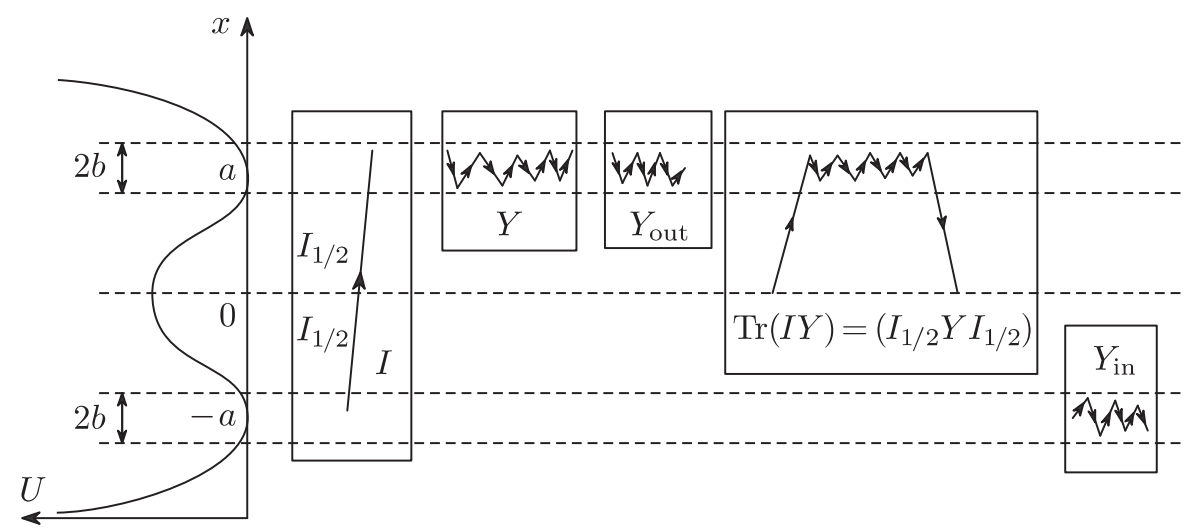

Через эти "блоки" амплитуду можно записать как (в операторной форме)

$$
i K_{E}(-a, a)=Y_{\text {in }} I Y_{\text {out }}+Y_{\text {in }} I Y I Y I Y_{\text {out }}+Y_{\text {in }} I Y I Y I Y I Y I Y_{\text {out }}+\cdots
$$

Заметим, что инстантонный оператор может быть факторизован: $I\left(x^{\prime \prime}, x^{\prime}\right)=$ $I_{1 / 2}\left(x^{\prime \prime}\right) I_{1 / 2}\left(x^{\prime}\right)$ или, в операторных терминах, $I=I_{1 / 2} I_{1 / 2}^{\mathrm{T}}$. Тогда

$$
\begin{aligned}
i K_{E}(-a, a) & =\left(Y_{\mathrm{in}} I_{1 / 2}\right)\left(I_{1 / 2}^{\mathrm{T}} Y_{\text {out }}\right)+\left(Y_{\text {in }} I_{1 / 2}\right)\left(I_{1 / 2}^{\mathrm{T}} Y I_{1 / 2}\right)\left(I_{1 / 2}^{\mathrm{T}} Y I_{1 / 2}\right)\left(I_{1 / 2}^{\mathrm{T}} Y_{\text {out }}\right)+\cdots= \\
& =\left(Y_{\text {in }} I_{1 / 2}\right) \sum_{n=0}^{\infty}(\operatorname{Tr} I Y)^{2 n}\left(I_{1 / 2}^{\mathrm{T}} Y_{\text {out }}\right)
\end{aligned}
$$

где каждое слагаемое в скобках является теперь просто скалярной функцией $E$ (не оператором). Умножение в энергетическом представлении соответствует свертке во временно́м представлении:

$$
\begin{aligned}
& A_{1}(E) A_{2}(E) \ldots A_{n+1}(E) \leftrightarrow \\
& \leftrightarrow \int_{\left\{t_{k+1}>t_{k}\right\}} \prod_{k=1}^{n} d t_{k} A_{n+1}\left(T-t_{n}\right) A_{n}\left(t_{n}-t_{n-1}\right) \ldots A_{1}\left(t_{1}\right) .
\end{aligned}
$$

При $A_{k}(t)=\mathrm{const} \cdot e^{-\alpha t}$ такая свертка пропорциональна $e^{-\alpha T} T^{n} / n !$. В нашем примере эта свертка в точности соответствует интегрированию по положениям центров инстантонов. Описанное в основном тексте квазиклассическое суммирование дает, что около самого левого полюса ${ }^{13)}$

$$
\operatorname{Tr} I Y \sim \frac{\hbar \omega / 2 \pi}{\hbar \omega / 2-E} e^{-S_{\mathrm{inst}} / \hbar} \Rightarrow(\operatorname{Tr} I Y)(T) \sim \frac{\omega}{2 \pi} e^{-\omega T / 2} e^{-S_{\mathrm{inst}} / \hbar}, \quad T \rightarrow \infty .
$$

Поэтому для больших $T$

$$
K_{T}(-a, a)=\sqrt{\frac{m \omega}{\pi \hbar}} e^{-\omega T / 2} \sum_{\substack{k=0 \\(k \equiv 1 \bmod 2)}}^{\infty} \frac{T^{k}}{k !}\left(\frac{\omega}{2 \pi}\right)^{k} e^{-k S_{\text {inst }}}=
$$

13) Здесь $S_{\text {inst }}$ совпадает с $R$, введенным выше. 


$$
=\sqrt{\frac{m \omega}{\pi \hbar}} e^{-\omega T / 2} \operatorname{sh}\left[T\left(\frac{\omega}{2 \pi}\right) e^{-S_{\mathrm{inst}}}\right] \Rightarrow \Delta E= \pm \frac{\hbar \omega}{2 \pi} e^{-S_{\mathrm{inst}} / \hbar}:
$$

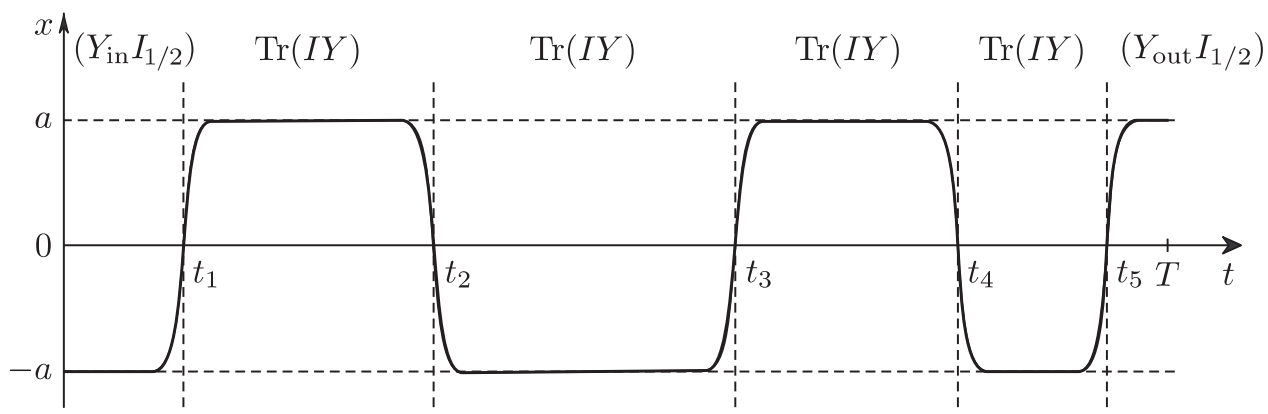

Благодарности. Автор благодарен А. Д. Миронову, А. Ю. Морозову и А. С. Лосеву за полезные вопросы и обсуждения. Работа частично поддержана Программой поддержки ведущих научных школ (грант № НШ-8065.2006.2) и РФФИ (грант № 04-02-16538).

\section{Список литературы}

[1] Р. Фейнман, А. Хиббс, Квантовая механика и интеграль по траекториям, Мир, М., 1968.

[2] Л. Д. Ландау, Е. М. Лившиц, Квантовая механика. Нерелятивистская теория, Наука, М., 1989.

[3] S. Coleman, "Uses of instantons", The Whys of Subnuclear Physics, Proceedings of the International School of Subnuclear Physics (Trapani, Sicily, July 23 - August 10, 1977), ed. A. Zichichi, Plenum, New York, 1979, 805.

[4] V. A. Novikov, M. A. Shifman, A. I. Vainshtein, V. I. Zakharov, "ABC of instantons", ITEP Lectures on Particle Physics and Field Theory, v. 1, World Sci. Lecture Notes Phys., 62, ed. M. A. Shifman, World Scientific, Singapore, 1999, 201-299.

[5] A. Polyakov, $2 D$ Quantum Gravity and $S C$ at high $T_{c}$, Elsevier Science Publishers B.V., 1989.

[6] А. М. Поляков, Калибровочные поля и струны, ИТФ, Черноголовка, 1995.

Поступила в редакцию 5.04.2007 